\title{
Bi-Objective Optimal Control Modification Adaptive Control for Systems with Input Uncertainty
}

\author{
Nhan T. Nguyen* \\ NASA Ames Research Center, Moffett Field, CA 94035
}

\begin{abstract}
This paper presents a new model-reference adaptive control method based on a bi-objective optimal control formulation for systems with input uncertainty. A parallel predictor model is constructed to relate the predictor error to the estimation error of the control effectiveness matrix. In this work, we develop an optimal control modification adaptive control approach that seeks to minimize a bi-objective linear quadratic cost function of both the tracking error norm and predictor error norm simultaneously. The resulting adaptive laws for the parametric uncertainty and control effectiveness uncertainty are dependent on both the tracking error and predictor error, while the adaptive laws for the feedback gain and command feedforward gain are only dependent on the tracking error. The optimal control modification term provides robustness to the adaptive laws naturally from the optimal control framework. Simulations demonstrate the effectiveness of the proposed adaptive control approach.
\end{abstract}

\section{Introduction}

Adaptive control has been used with success in a number of flight control applications. In certain situations, fast adaptation is needed in order to improve tracking performance rapidly when a system is subject to large uncertainty such as structural damage to an aircraft that could cause rapid changes in system dynamics. In these situations, adaptive control needs to be able to adapt quickly by the use of a large adaptive gain in order to reduce the tracking error as fast as possible. However, the use of a large adaptive gain in adaptive control can result in high frequency oscillations which can excite unmodeled dynamics that could adversely affect stability of an adaptive law. ${ }^{1}$ Poor robustness to unmodeled dynamics, time delay, and exogenous disturbances due to high gain adaptive control is well-known. Thus, typically there exists a balance between stability and adaptation. A large adaptive gain generally can improve tracking performance but usually at the expense of robustness.

To address the lack of robustness of the standard model-reference adaptive control, the two well-known robust modification methods in adaptive control, namely; the $\sigma$ modification ${ }^{2}$ and $e$ modification, ${ }^{3}$ have been used extensively in adaptive control. Recent years have seen a surge in many new adaptive control methods such as the $\mathscr{L}_{1}$ adaptive control, ${ }^{4}$ adaptive loop recovery, ${ }^{5}$ Kalman filter adaptive control, ${ }^{6}$ derivative-free adaptive control, ${ }^{7}$ composite model-reference adaptive control, ${ }^{8}$ and optimal control modification; ${ }^{9}$ just to name a few. In terms of addressing fast adaptation, the $\mathscr{L}_{1}$ adaptive control has gained a considerable attention due to its ability to achieve robustness with fast adaptation for a given a priori bound on the uncertainty. The existence of theoretical bounds on the transient performance and time delay margin of the $\mathscr{L}_{1}$ adaptive control enables it to address one of the current challenges in verification and validation: the lack of theoretically justifiable metrics. ${ }^{10}$ One of the key features of the $\mathscr{L}_{1}$ adaptive control is the existence of a linear input-output mapping with fast adaptation which helps to address the problem with predictability of nonlinear control.

The optimal control modification has been developed using an optimal control framework to minimize the $\mathscr{L}_{2}$ norm of the tracking error bounded away from the origin by some lower bound. ${ }^{9}$ By increasing the lower bound, robustness can be improved by trading off with tracking performance. This adaptive control method in some way can be shown to possess a certain degree of similarity to the $\mathscr{L}_{1}$ adaptive control with respect to fast adaptation. In particular, the optimal control modification can be shown to have an asymptotic linearity between inputs and outputs under the fast adaptation assumption. ${ }^{11,12,14}$ Hence, stability robustness of the optimal control modification can easily be analyzed.

A number of extensions have been developed for the optimal control modification method. In the presence of actuator rate limiting, a time-scale separation principle is applied to the method to decouple the slow-fast system via

${ }^{*}$ Research Scientist, Intelligent Systems Division, Mail Stop 269-1, AIAA Associate Fellow 
the singular perturbation. ${ }^{13}$ This approach improves tracking performance in the presence of slow actuator dynamics. For problems with control input uncertainty that limits the control effectiveness, a state predictor method has been developed for the optimal control modification to accommodate both the control input uncertainty and matched uncertainty. ${ }^{14}$ In the presence of linear matched uncertainty with fast adaptation, the optimal control modification method has been observed to exhibit an asymptotic linear property which has been further exploited to develop an analytical method for estimating a lower bound of the time delay margin for a given a priori information on the parametric uncertainty. ${ }^{11,12,14}$ The optimal control modification method has also been used in conjunction with the newly developed derivative-free adaptive control.

In terms of applications and validation, the optimal control modification method has been demonstrated in many flight environments ranging from low-fidelity desktop simulations, to medium-fidelity motion-based flight simulator experiments, and to the ultimate high-fidelity flight testing on a piloted aircraft. For desktop simulations, the optimal control modification method has been applied to various aircraft models including NASA Generic Transport Model (GTM) with damaged flight dynamics ${ }^{9}$ and aeroelastic longitudinal dynamics, ${ }^{16}$ a general aviation aircraft, ${ }^{17}$ and a NASA F/A-18A aircraft model. ${ }^{18}$ For medium-fidelity validation, a pilot evaluation study has been conducted in a motion-base flight simulator at NASA Ames Research Center in 2009 by eight NASA test pilots. Favorable CooperHarper ratings by the NASA test pilots have been noted with the optimal control modification adaptive law. ${ }^{19,20}$ For high-fidelity validation, a series of flight experiments have recently been conducted in late 2010 and early 2011 onboard a NASA F/A-18A test aircraft at NASA Dryden Flight Research Center to evaluate the effectiveness of the optimal control modification method. The flight test results show that the optimal control modification method offers the potential for flight control performance improvements under certain degraded flight control characteristics. ${ }^{21,22}$

In certain situations, the control effectiveness of a control system may be impaired due to failures. When an uncertainty exists in the control input, the system can undergo significant changes in its closed-loop characteristics that can compromise stability and performance of the control system. The control signal must be modified accordingly to produce achievable dynamics in the presence of the reduced control effectiveness. A new approach based on the optimal control modification adaptive law has been developed to address this issue. A parallel predictor model is constructed to relate the predictor error to the estimation error of the control effectiveness matrix. In this work, we will develop an optimal control modification adaptive control approach that seeks to minimize a bi-objective linear quadratic cost function of both the tracking error norm and predictor error norm simultaneously. The resulting adaptive laws for the parametric uncertainty and control effectiveness uncertainty are dependent on both the tracking error and predictor error, while the adaptive laws for the feedback gain and command feedforward gain are only dependent on the tracking error. In this context, the new adaptive law is similar to the composite model-reference adaptive control, ${ }^{8}$ but the difference lies in the optimal control modification term that provides robustness to the adaptive laws naturally from the optimal control formulation.

\section{Bi-Objective Optimal Control Modification Adaptive Laws}

Consider the following nonlinear system with control input uncertainty, match uncertainty, and unmatched disturbance

$$
\dot{x}=A x+B \Lambda\left[u+\Theta^{* \top} \Phi(x)\right]+w
$$

where $x(t) \in \mathbb{R}^{n}$ is a state vector, $u(t) \in \mathbb{R}^{m}$ is a control vector, $A \in \mathbb{R}^{n} \times \mathbb{R}^{n}$ is known, $B \in \mathbb{R}^{n} \times \mathbb{R}^{m}$ is also known such that $(A, B)$ is controllable, $\Lambda=\Lambda^{\top}>0 \in \mathbb{R}^{m} \times \mathbb{R}^{m}$ is a constant unknown diagonal matrix with diagonal elements that represents a control input uncertainty, $\Theta^{*} \in \mathbb{R}^{p} \times \mathbb{R}^{m}$ is a constant and unknown matrix that represents a matched parametric uncertainty, $\Phi(x) \in \mathbb{R}^{p}$ is a known structure of the match uncertainty, and $w(t) \in \mathbb{R}^{n}$ is an unmatched bounded disturbance with bounded time derivative, i.e., $\sup _{\forall t}\|w\| \leq w_{0}$ and $\sup _{\forall t}\|\dot{w}\| \leq \dot{w}_{0}$.

A nominal fixed gain controller is designed to stabilize the nominal plant with no uncertainty, i.e., $\Lambda=I$ and $\Theta^{*}=0$, and to enable it to track a reference command signal $r(t)$ as follows:

$$
\bar{u}=K_{x} x+K_{r} r
$$

where $r(t) \in \mathbb{R}^{r}$ is a bounded reference command signal, such that $A+B K_{x} \in \mathbb{R}^{n \times n}$ is Hurwitz, and $B K_{r} \in \mathbb{R}^{n \times r}$.

The closed-loop nominal plant without uncertainty is then used to specify a reference model

$$
\dot{x}_{m}=A_{m} x_{m}+B_{m} r
$$

where $x_{m}(t) \in \mathbb{R}^{n}$ is a reference state vector, $A_{m}=A+B K_{x}$, and $B_{m}=B K_{r}$. 
In the presence of both the control input uncertainty and matched uncertainty due to $\Lambda$ and $\Theta^{*}$, an adaptive controller is designed as

$$
u=K_{x}(t) x+K_{r}(t) r-\Theta^{\top}(t) \Phi(x)
$$

where $K_{x}(t) \in \mathbb{R}^{m} \times \mathbb{R}^{n}$ is an adaptive feedback gain, $K_{r}(t) \in \mathbb{R}^{m} \times \mathbb{R}^{r}$ is an adaptive command feedforward gain, and $\Theta(t) \in \mathbb{R}^{p} \times \mathbb{R}^{m}$ is the estimate of $\Theta^{*}$.

We assume that there exist constant and unknown matrices $K_{x}^{*}$ and $K_{r}^{*}$ such that the following matching conditions are satisfied

$$
\begin{aligned}
& \Lambda K_{x}^{*}=K_{x} \\
& \Lambda K_{r}^{*}=K_{r}
\end{aligned}
$$

If $\Lambda$ is unknown but sign of $\Lambda$ is known, then the standard MRAC adaptive laws are given by

$$
\begin{gathered}
\dot{K}_{x}^{\top}=\Gamma_{x} x e^{\top} P B \operatorname{sgn} \Lambda \\
\dot{K}_{r}^{\top}=\Gamma_{r} r e^{\top} P B \operatorname{sgn} \Lambda \\
\dot{\Theta}=-\Gamma_{\Theta} \Phi(x) e^{\top} P B \operatorname{sgn} \Lambda
\end{gathered}
$$

It is well-known that the standard MRAC is non-robust. To improve robustness, the adaptive laws should include a robust modification scheme or the projection method. If $\Lambda$ is completely unknown. Then, we need to consider other approaches. We now introduce an optimal control modification method that uses two types of errors for adaptation: tracking error and predictor error. We call this bi-objective optimal control modification adaptive control.

Let $\tilde{\Lambda}(t)=\hat{\Lambda}(t)-\Lambda, \tilde{K}_{x}(t)=K_{x}(t)-K_{x}^{*}, \tilde{K}_{r}(t)=K_{r}(t)-K_{r}^{*}$, and $\tilde{\Theta}(t)=\Theta(t)-\Theta^{*}$ be the estimation errors. Then the closed-loop plant becomes

$$
\dot{x}=A_{m} x+B_{m} r+B(\hat{\Lambda}-\tilde{\Lambda})\left[\tilde{K}_{x} x+\tilde{K}_{r} r-\tilde{\Theta}^{\top} \Phi(x)\right]+w
$$

Then the tracking error equation becomes

$$
\dot{e}=A_{m} e+B \hat{\Lambda}\left[-\tilde{K}_{x} x-\tilde{K}_{r} r+\tilde{\Theta}^{\top} \Phi(x)\right]-w+B \varepsilon
$$

where $\varepsilon(x, r) \in \mathbb{R}^{m}$ is the residual estimation error of the plant model

$$
\varepsilon=\tilde{\Lambda}\left[\tilde{K}_{x} x+\tilde{K}_{r} r-\tilde{\Theta}^{\top} \Phi(x)\right]
$$

such that $\sup _{\forall x, r}\|\varepsilon\| \leq \varepsilon_{0}$.

Consider a predictor model of the plant as

$$
\dot{\hat{x}}=A_{m} \hat{x}+\left(A-A_{m}\right) x+B \hat{\Lambda}\left[u+\Theta^{\top} \Phi(x)\right]+\hat{w}
$$

where $\hat{w}(t)$ is the estimate of the disturbance $w(t)$.

We define the predictor error as $e_{p}(t)=\hat{x}(t)-x(t)$, then

$$
\dot{e}_{p}=A_{m} e_{p}+B \tilde{\Lambda}\left[u+\Theta^{\top} \Phi(x)\right]+B \hat{\Lambda} \tilde{\Theta}^{\top} \Phi(x)+\tilde{w}+B \varepsilon_{p}
$$

where $\tilde{w}(t)=\hat{w}(t)-w(t)$ is the disturbance estimation error, and $\varepsilon_{p}(x) \in \mathbb{R}^{m}$ is the residual estimation error of the predictor model

$$
\varepsilon_{p}=-\tilde{\Lambda} \tilde{\Theta}^{\top} \Phi(x)
$$

such that $\sup _{\forall x}\left\|\varepsilon_{p}\right\| \leq \varepsilon_{p_{0}}$.

Proposition: The bi-objective optimal control modification adaptive laws are defined as:

$$
\begin{aligned}
& \dot{K}_{x}^{\top}=\Gamma_{x} x\left(e^{\top} P+v u^{\top} \hat{\Lambda}^{\top} B^{\top} P A_{m}^{-1}\right) B \hat{\Lambda} \\
& \dot{K}_{r}^{\top}=\Gamma_{r} r\left(e^{\top} P+v u^{\top} \hat{\Lambda}^{\top} B^{\top} P A_{m}^{-1}\right) B \hat{\Lambda}
\end{aligned}
$$




$$
\begin{gathered}
\dot{\Theta}=-\Gamma_{\Theta} \Phi(x)\left(e^{\top} P+v u^{\top} \hat{\Lambda}^{\top} B^{\top} P A_{m}^{-1}+e_{p}^{\top} W-\eta\left\{\left[u+2 \Theta^{\top} \Phi(x)\right]^{\top} \hat{\Lambda}^{\top} B^{\top}+\hat{w}^{\top}\right\} W A_{m}^{-1}\right) B \hat{\Lambda} \\
\dot{\hat{\Lambda}}^{\top}=-\Gamma_{\Lambda}\left[u+\Theta^{\top} \Phi(x)\right]\left(e_{p}^{\top} W-\eta\left\{\left[u+2 \Theta^{\top} \Phi(x)\right]^{\top} \hat{\Lambda}^{\top} B^{\top}+\hat{w}^{\top}\right\} W A_{m}^{-1}\right) B \\
\dot{\hat{w}}^{\top}=-\gamma_{w}\left(e_{p}^{\top} W-\eta\left\{\left[u+2 \Theta^{\top} \Phi(x)\right]^{\top} \hat{\Lambda}^{\top} B^{\top}+\hat{w}^{\top}\right\} W A_{m}^{-1}\right)
\end{gathered}
$$

where $\Gamma_{x}=\Gamma_{x}^{\top}>0 \in \mathbb{R}^{n} \times \mathbb{R}^{n}, \Gamma_{r}=\Gamma_{r}^{\top}>0 \in \mathbb{R}^{r} \times \mathbb{R}^{r}, \Gamma_{\Theta}=\Gamma_{\Theta}^{\top}>0 \in \mathbb{R}^{m} \times \mathbb{R}^{m}, \Gamma_{\Lambda}=\Gamma_{\Lambda}^{\top}>0 \in \mathbb{R}^{p} \times \mathbb{R}^{p}$, and $\gamma_{w}>0 \in \mathbb{R}$ are adaptation rate matrices; $v>0 \in \mathbb{R}$ and $\eta>0 \in \mathbb{R}$ are the optimal control modification parameters; and $P=P^{\top}>0 \in \mathbb{R}^{n \times n}$ and $W=W^{\top}>0 \in \mathbb{R}^{n \times n}$ are solutions of the following Lyapunov equations

$$
\begin{gathered}
P A_{m}+A_{m}^{\top} P=-Q \\
W A_{m}+A_{m}^{\top} W=-R
\end{gathered}
$$

where $Q=Q^{\top}>0 \in \mathbb{R}^{n \times n}$ and $R=R^{\top}>0 \in \mathbb{R}^{n \times n}$ are positive definite weighting matrices.

We note that $K_{x}$ and $K_{r}$ are adapted based on the tracking error, $\hat{\Lambda}$ and $\hat{w}$ are estimated using the predictor error, and $\Theta$ is adapted based on both the tracking error and predictor error.

The adaptive control architecture with the bi-objective optimal control modification is presented in Figure 1.

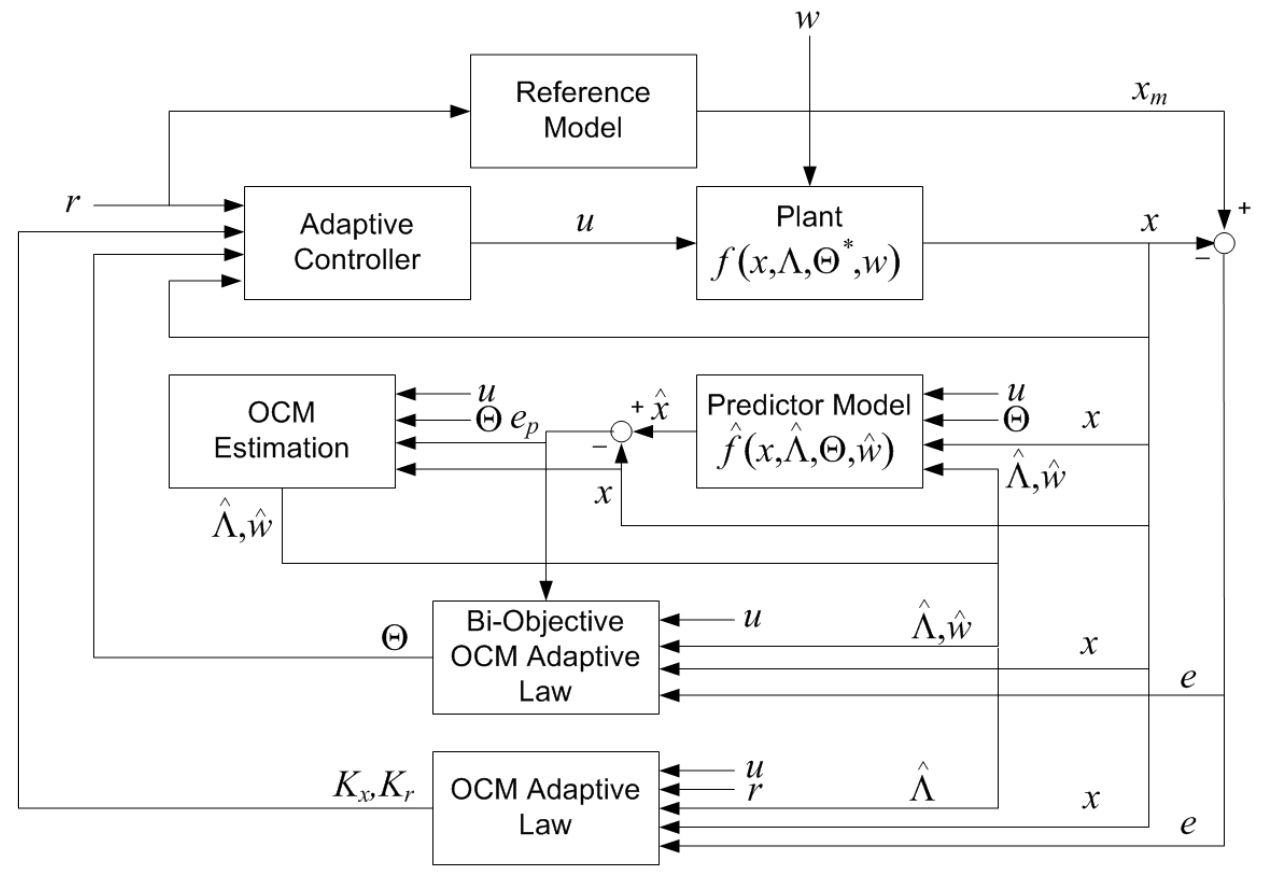

Figure 1 - Adaptive Control Architecture with Bi-Objective Optimal Control Modification

Proof: The optimal control modification adaptive laws are called bi-objective because they use both the tracking error and predictor error for adaptation and are derived from the following infinite-time horizon cost functions

$$
\begin{aligned}
& J_{1}=\lim _{t_{f} \rightarrow \infty} \frac{1}{2} \int_{0}^{t_{f}}\left(e-\Delta_{1}\right)^{\top} Q\left(e-\Delta_{1}\right) d t \\
& J_{2}=\lim _{t_{f} \rightarrow \infty} \frac{1}{2} \int_{0}^{t_{f}}\left(e_{p}-\Delta_{2}\right)^{\top} R\left(e_{p}-\Delta_{2}\right) d t
\end{aligned}
$$

subject to Eqs. (11) and (14), where $\Delta_{1}$ and $\Delta_{2}$ represent the unknown lower bounds of the tracking error and the predictor error, respectively.

The cost functions $J_{1}$ and $J_{2}$ are combined into the following bi-objective cost function

$$
J=J_{1}+J_{2}
$$


The bi-objective cost function $J$ combines both the objectives of minimization of the tracking error and the predictor error bounded away from the origin. Geometrically, it represents a distance measured from a point on the trajectory of $e(t)$ and $e_{p}(t)$ to the normal surface of a hypersphere $B_{\Delta}=\left\{e(t) \in \mathbb{R}^{n}, e_{p}(t) \in \mathbb{R}^{n}:\left(e-\Delta_{1}\right)^{\top} Q\left(e-\Delta_{1}\right)\right.$ $\left.+\left(e_{p}-\Delta_{2}\right)^{\top} R\left(e_{p}-\Delta_{2}\right) \leq 0\right\} \subset \mathscr{D} \subset \mathbb{R}^{n}$. The bi-objective cost function is designed to provide stability robustness by not seeking asymptotic tracking and predictor errors that tend to zero as $t_{f} \rightarrow \infty$, but rather errors that tend to some lower bound away from the origin. By not requiring asymptotic tracking and predictor errors, the adaptation can be made more robust. Therefore, the tracking performance can be traded with stability robustness by a suitable selection of the modification parameters $v$ and $\eta$. Increasing the tracking performance by reducing $v$ and or $\eta$ will decrease stability robustness of the adaptive laws to unmodeled dynamics, and vice versa.

The derivation of the adaptive laws is established by the Pontryagin's Minimum Principle. Using the optimal control framework, the Hamiltonian of the cost function is defined as

$$
\begin{array}{r}
H=\frac{1}{2}\left(e-\Delta_{1}\right)^{\top} Q\left(e-\Delta_{1}\right)+\frac{1}{2}\left(e_{p}-\Delta_{2}\right)^{\top} R\left(e_{p}-\Delta_{2}\right)+\lambda^{\top}\left\{A_{m} e+B \hat{\Lambda}\left[-\tilde{K}_{x} x-\tilde{K}_{r} r+\tilde{\Theta}^{\top} \Phi(x)\right]-w+B \varepsilon\right\} \\
+\mu^{\top}\left\{A_{m} e_{p}+B \tilde{\Lambda}\left[u+\Theta^{\top} \Phi(x)\right]+B \hat{\Lambda} \tilde{\Theta}^{\top} \Phi(x)+\tilde{w}+B \varepsilon_{p}\right\}
\end{array}
$$

where $\lambda(t):[0, \infty) \rightarrow \mathbb{R}^{n}$ and $\mu(t):[0, \infty) \rightarrow \mathbb{R}^{n}$ are adjoint variables.

The adjoint equations can be obtained from the necessary conditions of optimality as

$$
\begin{aligned}
& \dot{\lambda}=-\nabla H_{e}^{\top}=-Q\left(e-\Delta_{1}\right)-A_{m}^{\top} \lambda \\
& \dot{\mu}=-\nabla H_{e_{p}}^{\top}=-R\left(e_{p}-\Delta_{2}\right)-A_{m}^{\top} \mu
\end{aligned}
$$

subject to the transversality conditions $\lambda\left(t_{f} \rightarrow \infty\right)=0$ and $\mu\left(t_{f} \rightarrow \infty\right)=0$ since both $e(0)$ and $e_{p}(0)$ are known.

Treating $\tilde{K}_{x}, \tilde{K}_{r}, \tilde{\Theta}(t), \tilde{\Lambda}$, and $\tilde{w}$ as control variables, then the optimal control solutions are obtained by the following gradient-based adaptive laws:

$$
\begin{gathered}
\dot{K}_{x}^{\top}=\dot{\tilde{K}}_{x}^{\top}=-\Gamma_{x} \nabla H_{\tilde{K}_{x}}=\Gamma_{x} x \lambda^{\top} B \hat{\Lambda} \\
\dot{K}_{r}^{\top}=\dot{\tilde{K}}_{r}^{\top}=-\Gamma_{r} \nabla H_{\tilde{K}_{r}}=\Gamma_{r} r \lambda^{\top} B \hat{\Lambda} \\
\dot{\Theta}=\dot{\tilde{\Theta}}=-\Gamma_{\Theta} \nabla H_{\tilde{\Theta}}^{\top}=-\Gamma_{\Theta} \Phi(x)\left(\lambda^{\top}+\mu^{\top}\right) B \hat{\Lambda} \\
\dot{\hat{\Lambda}}^{\top}=\dot{\tilde{\Lambda}}^{\top}=-\Gamma_{\Lambda} \nabla H_{\tilde{\Lambda}}=-\Gamma_{\Lambda}\left[u+\Theta^{\top} \Phi(x)\right] \mu^{\top} B \\
\dot{\hat{w}}^{\top}=-\gamma_{w} \nabla H_{\tilde{w}}=-\gamma_{w} \mu^{\top}
\end{gathered}
$$

The closed-form solutions can be obtained by eliminating the adjoint variables $\lambda(t)$ and $\mu(t)$ using the "sweep" method with the following assumed solutions of the adjoint equations

$$
\begin{gathered}
\lambda=P e+S\left[-K_{x} x-K_{r} r+\Theta^{\top} \Phi(x)\right] \\
\mu=W e_{p}+T\left[u+2 \Theta^{\top} \Phi(x)\right]+V
\end{gathered}
$$

Substituting the adjoint solutions back into the adjoint equations yields

$$
\begin{aligned}
& \dot{P} e+P A_{m} e+ P B \hat{\Lambda}\left[-K_{x} x-K_{r} r+\Theta^{\top} \Phi(x)\right]-P B \hat{\Lambda}\left[-K_{x}^{*} x-K_{r}^{*} r+\Theta^{* \top} \Phi(x)\right]-P w+P B \varepsilon \\
&+ \dot{S}\left[-K_{x} x-K_{r} r+\Theta^{\top} \Phi(x)\right]+S \frac{d\left[-K_{x} x-K_{r} r+\Theta^{\top} \Phi(x)\right]}{d t}=-Q\left(e-\Delta_{1}\right)-A_{m}^{\top} P e \\
&-A_{m}^{\top} S\left[-K_{x} x-K_{r} r+\Theta^{\top} \Phi(x)\right] \\
& \dot{W} e_{p}+W A_{m} e_{p}+W B \hat{\Lambda}\left[u+\Theta^{\top} \Phi(x)\right]-W B \Lambda\left[u+\Theta^{\top} \Phi(x)\right]+W B \hat{\Lambda} \Theta^{\top} \Phi(x)-W B \hat{\Lambda} \Theta^{* \top} \Phi(x)+W \hat{w}-W w \\
&+W B \varepsilon_{p}+\dot{T}\left[u+2 \Theta^{\top} \Phi(x)\right]+T \frac{d\left[u+2 \Theta^{\top} \Phi(x)\right]}{d t}+\dot{V}=-R\left(e_{p}-\Delta_{2}\right)-A_{m}^{\top} W e_{p} \\
&-A_{m}^{\top} T\left[u+2 \Theta^{\top} \Phi(x)\right]-A_{m}^{\top} V
\end{aligned}
$$


Equating terms yields the following equations

$$
\begin{gathered}
\dot{P}+P A_{m}+A_{m}^{\top} P+Q=0 \\
\dot{S}+A_{m}^{\top} S+P B \hat{\Lambda}=0 \\
Q \Delta_{1}+P B \hat{\Lambda}\left[-K_{x}^{*} x-K_{r}^{*} r+\Theta^{* \top} \Phi(x)\right]+P w-P B \varepsilon-S \frac{d\left[-K_{x} x-K_{r} r+\Theta^{\top} \Phi(x)\right]}{d t}=0 \\
\dot{W}+W A_{m}+A_{m}^{\top} W+R=0 \\
\dot{T}+A_{m}^{\top} T+W B \hat{\Lambda}=0 \\
\dot{V}+A_{m}^{\top} V+W \hat{w}=0 \\
R \Delta_{2}+W B \Lambda\left[u+\Theta^{\top} \Phi(x)\right]+W B \hat{\Lambda} \Theta^{* \top} \Phi(x)+W w-W B \varepsilon_{p}-T \frac{d\left[u+2 \Theta^{\top} \Phi(x)\right]}{d t}-\dot{V}=0
\end{gathered}
$$

subject to the transversality conditions $P\left(t_{f} \rightarrow \infty\right)=0, S\left(t_{f} \rightarrow \infty\right)=0, W\left(t_{f} \rightarrow \infty\right)=0$, and $T\left(t_{f} \rightarrow \infty\right)=0$.

The existence and uniqueness of the solution of the Lyapunov differential equations. The infinite-time horizon solutions of $P(t)$ and $W(t)$ as $t_{f} \rightarrow \infty$ of the Lyapunov differential equations tend to their equilibrium solutions at $t=0$

$$
\begin{gathered}
P A_{m}+A_{m}^{\top} P+Q=0 \\
W A_{m}+A_{m}^{\top} W+R=0
\end{gathered}
$$

and the solutions of $S(t), T(t)$, and $V(t)$ tend to their equilibrium solutions

$$
\begin{gathered}
A_{m}^{\top} S+P B \hat{\Lambda}=0 \\
A_{m}^{\top} T+W B \hat{\Lambda}=0 \\
A_{m}^{\top} V+W \hat{w}=0
\end{gathered}
$$

As with any control design, performance and robustness are often considered as two competing design requirements. Increasing robustness tends to require a compromise in performance and vice versa. Thus, to enable the bi-objective optimal control modification adaptive laws to be sufficiently flexible for control design, the modification parameters $v>0$ and $\eta>0$ are introduced as design free parameters to allow for adjustments of the optimal control modification terms in the adaptive laws.

Thus, the solutions of $S(t), T(t)$, and $V(t)$ are given by

$$
\begin{gathered}
S=-v A_{m}^{-\top} P B \hat{\Lambda} \\
T=-\eta A_{m}^{-\top} W B \hat{\Lambda} \\
V=-\eta A_{m}^{-\top} W \hat{w}
\end{gathered}
$$

Using the expression of $u(t)$, the adjoint solutions are then obtained as

$$
\begin{gathered}
\lambda=P e+v A_{m}^{-\top} P B \hat{\Lambda} u \\
\mu=W e_{p}-\eta A_{m}^{-\top} W B \hat{\Lambda}\left[u+2 \Theta^{\top} \Phi(x)\right]-\eta A_{m}^{-\top} W \hat{w}
\end{gathered}
$$

Substituting the adjoint solutions into the gradient-based adaptive laws yields the bi-objective optimal control modification adaptive laws.

The bounds on $\Delta_{1}(t)$ and $\Delta_{2}(t)$ as $t_{f} \rightarrow \infty$ are given by

$$
\begin{aligned}
\left\|\Delta_{1}\right\| \leq \frac{1}{\lambda_{\min }(Q)}\left[\|P B \hat{\Lambda}\|\left\|-K_{x}^{*} x-\Delta K_{r}^{*} r+\Theta^{* \top} \Phi(x)\right\|+\right. & \lambda_{\max }(P) w_{0}+\|P B\| \varepsilon_{0} \\
& \left.+v\left\|A_{m}^{-\top} P B \hat{\Lambda}\right\|\left\|\frac{d\left[-K_{x} x-K_{r} r+\Theta^{\top} \Phi(x)\right]}{d t}\right\|\right]
\end{aligned}
$$




$$
\begin{array}{r}
\left\|\Delta_{2}\right\| \leq \frac{1}{\lambda_{\min }(R)}\left[\|W B \Lambda\|\left\|u+\Theta^{\top} \Phi(x)\right\|+\|W B \hat{\Lambda}\|\left\|\Theta^{* \top} \Phi(x)\right\|+\lambda_{\max }(W) w_{0}+\|W B\| \varepsilon_{p_{0}}\right. \\
\left.+\eta\left\|A_{m}^{-\top} W B\right\|\left\|\frac{d\left[u+\Theta^{\top} \Phi(x)\right]}{d t}\right\|\right]
\end{array}
$$

which are dependent upon the modification parameters, control effectiveness uncertainty, matched uncertainty, unmatched disturbance, and residual tracking error and predictor error.

Note that if $R=Q$ and $\eta=v$, then the bi-objective optimal control modification adaptive laws for $\Theta(t), \hat{\Lambda}(t)$, and $\hat{w}(t)$ become

$$
\begin{gathered}
\dot{\Theta}=-\Gamma_{\Theta} \Phi(x)\left(e^{\top} P+e_{p}^{\top} P-v\left\{2 \Phi^{\top}(x) \Theta \hat{\Lambda}^{\top} B^{\top}+\hat{w}^{\top}\right\} P A_{m}^{-1}\right) B \hat{\Lambda} \\
\dot{\hat{\Lambda}}^{\top}=-\Gamma_{\Lambda}\left[u+\Theta^{\top} \Phi(x)\right]\left(e_{p}^{\top} P-v\left\{\left[u+2 \Theta^{\top} \Phi(x)\right]^{\top} \hat{\Lambda}^{\top} B^{\top}+\hat{w}^{\top}\right\} P A_{m}^{-1}\right) B \\
\dot{\hat{w}}^{\top}=-\gamma_{w}\left(e_{p}^{\top} P-v\left\{\left[u+2 \Theta^{\top} \Phi(x)\right]^{\top} \hat{\Lambda}^{\top} B^{\top}+\hat{w}^{\top}\right\} P A_{m}^{-1}\right)
\end{gathered}
$$

Theorem: The bi-objective optimal control modification adaptive laws result in stable and uniformly ultimately bounded tracking error $e(t)$ and predictor error $e_{p}(t)$.

Proof: Choose a Lyapunov candidate function

$$
\begin{aligned}
V\left(e, e_{p}, \tilde{K}_{x}, \tilde{K}_{r}, \tilde{\Theta}, \tilde{\Lambda}, \tilde{w}\right)=e^{\top} P e+e_{p}^{\top} W e_{p}+\operatorname{trace}\left(\tilde{K}_{x} \Gamma_{x}^{-1} \tilde{K}_{x}^{\top}\right)+\operatorname{trace}\left(\tilde{K}_{r} \Gamma_{r}^{-1} \tilde{K}_{r}^{\top}\right) \\
+\operatorname{trace}\left(\tilde{\Theta}^{\top} \Gamma_{\Theta}^{-1} \tilde{\Theta}\right)+\operatorname{trace}\left(\tilde{\Lambda} \Gamma_{\Lambda}^{-1} \tilde{\Lambda}^{\top}\right)+\tilde{w}^{\top} \gamma_{w}^{-1} \tilde{w}^{\prime}
\end{aligned}
$$

Evaluating $\dot{V}\left(e, e_{p}, \tilde{K}_{x}, \tilde{K}_{r}, \tilde{\Theta}, \tilde{\Lambda}, \tilde{w}\right)$ yields

$$
\begin{aligned}
\dot{V}=e^{\top}( & \left.P A_{m}+A_{m}^{\top} P\right) e+2 e^{\top} P B \hat{\Lambda}\left[-\tilde{K}_{x} x-\tilde{K}_{r} r+\tilde{\Theta}^{\top} \Phi(x)\right]-2 e^{\top} P w+2 e^{\top} P B \varepsilon \\
& +e_{p}^{\top}\left(W A_{m}+A_{m}^{\top} W\right) e_{p}+2 e_{p}^{\top} W B\left\{\tilde{\Lambda}\left[u+\Theta^{\top} \Phi(x)\right]+\hat{\Lambda}^{\top} \Phi(x)\right\}+2 e_{p}^{\top} W \tilde{w}+2 e_{p}^{\top} W B \varepsilon_{p} \\
& +2 \operatorname{trace}\left(\tilde{K}_{x} x\left(e^{\top} P+v u^{\top} \hat{\Lambda}^{\top} B^{\top} P A_{m}^{-1}\right) B \hat{\Lambda}\right)+2 \operatorname{trace}\left(\tilde{K}_{r} r\left(e^{\top} P+v u^{\top} \hat{\Lambda}^{\top} B^{\top} P A_{m}^{-1}\right) B \hat{\Lambda}\right) \\
- & 2 \operatorname{trace}\left(\tilde{\Theta}^{\top} \Phi(x)\left(e^{\top} P+v u^{\top} \hat{\Lambda}^{\top} B^{\top} P A_{m}^{-1}+e_{p}^{\top} W-\eta\left\{\left[u+2 \Theta^{\top} \Phi(x)\right]^{\top} \hat{\Lambda}^{\top} B^{\top}+\hat{w}^{\top}\right\} W A_{m}^{-1}\right) B \hat{\Lambda}\right) \\
- & 2 \operatorname{trace}\left(\tilde{\Lambda}\left[u+\Theta^{\top} \Phi(x)\right]\left(e_{p}^{\top} W-\eta\left\{\left[u+2 \Theta^{\top} \Phi(x)\right]^{\top} \hat{\Lambda}^{\top} B^{\top}+\hat{w}^{\top}\right\} W A_{m}^{-1}\right) B\right)-2 e_{p}^{\top} W \tilde{w} \\
& +2\left(\eta\left\{\left[u+2 \Theta^{\top} \Phi(x)\right]^{\top} \hat{\Lambda}^{\top} B^{\top}+\hat{w}^{\top}\right\} W A_{m}^{-1}\right) \tilde{w}-2 \dot{w}^{\top} \gamma_{w}^{-1} \tilde{w}
\end{aligned}
$$

Using the trace identity trace $\left(A^{\top} B\right)=B A^{\top}, \dot{V}\left(e, e_{p}, \tilde{K}_{x}, \tilde{K}_{r}, \tilde{\Theta}, \tilde{\Lambda}, \tilde{w}\right)$ can be further simplified as

$$
\begin{aligned}
\dot{V}=-e^{\top} Q e-2 e^{\top} P w+2 e^{\top} P B \varepsilon-e_{p}^{\top} R e_{p}+2 e_{p}^{\top} W B \varepsilon_{p}-2 \dot{w}^{\top} \gamma_{w}^{-1} \tilde{w} \\
+2 v u^{\top} \hat{\Lambda}^{\top} B^{\top} P A_{m}^{-1} B \hat{\Lambda} \tilde{u}+2 \eta \operatorname{trace}\left(\tilde{\Theta}^{\top} \Phi(x)\left\{\left[u+2 \Theta^{\top} \Phi(x)\right]^{\top} \hat{\Lambda}^{\top} B^{\top}+\hat{w}^{\top}\right\} W A_{m}^{-1} B \hat{\Lambda}\right) \\
+2 \eta \operatorname{trace}\left(\tilde{\Lambda}\left[u+\Theta^{\top} \Phi(x)\right]\left\{\left[u+2 \Theta^{\top} \Phi(x)\right]^{\top} \hat{\Lambda}^{\top} B^{\top}+\hat{w}^{\top}\right\} W A_{m}^{-1} B\right) \\
+2 \eta \operatorname{trace}\left(\tilde{w}\left\{\left[u+2 \Theta^{\top} \Phi(x)\right]^{\top} \hat{\Lambda}^{\top} B^{\top}+\hat{w}^{\top}\right\} W A_{m}^{-1}\right)
\end{aligned}
$$

where $\tilde{u}=\tilde{K}_{x} x+\tilde{K}_{r} r-\tilde{\Theta} \Phi(x)$. 
Let $\bar{B}=\left[\begin{array}{ccc}B \hat{\Lambda} & B & I\end{array}\right] \in \mathbb{R}^{n} \times \mathbb{R}^{2 m+n}, \Omega=\left[\begin{array}{ccc}\Theta & 0 & 0 \\ 0 & \hat{\Lambda}^{\top} & 0 \\ 0 & 0 & \hat{w}^{\top}\end{array}\right] \in \mathbb{R}^{p+m+1} \times \mathbb{R}^{2 m+n}, \Psi(x, r)=\left[\begin{array}{c}\Phi(x) \\ u+\Theta^{\top} \Phi(x) \\ 1\end{array}\right] \in$ $\mathbb{R}^{p+m+1}$. Then

$$
\begin{array}{r}
\operatorname{trace}\left(\tilde{\Omega}^{\top} \Psi(x, r) \Psi^{\top}(x, r) \Omega \bar{B}^{\top} W A_{m}^{-1} \bar{B}\right)=\operatorname{trace}\left(\tilde{\Theta}^{\top} \Phi(x)\left\{\left[u+2 \Theta^{\top} \Phi(x)\right]^{\top} \hat{\Lambda}^{\top} B^{\top}+\hat{w}^{\top}\right\} W A_{m}^{-1} B \hat{\Lambda}\right) \\
\operatorname{trace}\left(\tilde{\Lambda}\left[u+\Theta^{\top} \Phi(x)\right]\left\{\left[u+2 \Theta^{\top} \Phi(x)\right]^{\top} \hat{\Lambda}^{\top} B^{\top}+\hat{w}^{\top}\right\} W A_{m}^{-1} B\right) \\
+\operatorname{trace}\left(\tilde{w}\left\{\left[u+2 \Theta^{\top} \Phi(x)\right]^{\top} \hat{\Lambda}^{\top} B^{\top}+\hat{w}^{\top}\right\} W A_{m}^{-1}\right)
\end{array}
$$

Thus,

$$
\begin{aligned}
\dot{V}=-e^{\top} Q e-2 e^{\top} P w+2 e^{\top} P B \varepsilon-e_{p}^{\top} R e_{p}+ & 2 e_{p}^{\top} W B \varepsilon_{p}-2 \dot{w}^{\top} \gamma_{w}^{-1} \tilde{w} \\
& +2 v u^{\top} \hat{\Lambda}^{\top} B^{\top} P A_{m}^{-1} B \hat{\Lambda} \tilde{u}+2 \eta \Psi^{\top}(x, r) \Omega \bar{B}^{\top} W A_{m}^{-1} \bar{B} \tilde{\Omega}^{\top} \Psi(x, r)
\end{aligned}
$$

Note that $P A_{m}^{-1}$ and $W A_{m}^{-1}$ are both negative definite matrices, therefore

$$
\begin{aligned}
& \dot{V}=-e^{\top} Q e-2 e^{\top} P w+2 e^{\top} P B \varepsilon-e_{p}^{\top} R e_{p}+2 e_{p}^{\top} W B \varepsilon_{p}-2 \dot{w}^{\top} \gamma_{w}^{-1} \tilde{w} \\
&-v \tilde{u}^{\top} \hat{\Lambda}^{\top} B^{\top} A_{m}^{-\top} Q A_{m}^{-1} B \hat{\Lambda} \tilde{u}-\eta \Psi^{\top}(x, r) \tilde{\Omega} \bar{B}^{\top} A_{m}^{-\top} R A_{m}^{-1} \bar{B} \tilde{\Omega}^{\top} \Psi(x, r) \\
&+2 v u^{* \top} \hat{\Lambda}^{\top} B^{\top} P A_{m}^{-1} B \hat{\Lambda} \tilde{u}+2 \eta \Psi^{\top}(x, r) \Omega^{*} \bar{B}^{\top} W A_{m}^{-1} \bar{B} \tilde{\Omega}^{\top} \Psi(x, r)
\end{aligned}
$$

Let $K=\left[\begin{array}{lll}K_{x} & K_{r} & -\Theta^{\top}\end{array}\right] \in \mathbb{R}^{m \times(n+r+p)}$ and $z(x, r)=\left[\begin{array}{c}x \\ r \\ \Phi(x)\end{array}\right] \in \mathbb{R}^{(n+r+p)}$. Then $u=K z(x, r)$ and

$$
\begin{array}{r}
\dot{V} \leq-\lambda_{\min }(Q)\|e\|^{2}+2\|e\| \lambda_{\max }(P) w_{0}+2\|e\|\|P B\| \varepsilon_{0}-\lambda_{\min }(R)\left\|e_{p}\right\|^{2}+2\left\|e_{p}\right\|\|W B\| \varepsilon_{p_{0}} \\
+2 \gamma_{w}^{-1}\|\tilde{\Omega}\| \dot{w}_{0}-v \lambda_{\min }\left(B^{\top} A_{m}^{-\top} Q A_{m}^{-1} B\right)\|z(x, r)\|^{2}\|\hat{\Lambda}\|^{2}\|\tilde{K}\|^{2}+2 v\|z(x, r)\|^{2}\left\|B^{\top} P A_{m}^{-1} B\right\|\|\hat{\Lambda}\|^{2}\|\tilde{K}\| K_{0} \\
-\eta \lambda_{\min }\left(A_{m}^{-\top} R A_{m}^{-1}\right)\|\Psi(x, r)\|^{2}\|\bar{B}\|^{2}\|\tilde{\Omega}\|^{2}+2 \eta\left\|W A_{m}^{-1}\right\|\|\Psi(x, r)\|^{2}\|\bar{B}\|^{2}\|\tilde{\Omega}\| \Omega_{0}
\end{array}
$$

Let $c_{1}=\lambda_{\text {min }}(Q), c_{2}=\frac{\lambda_{\max }(P) w_{0}+\|P B\| \varepsilon_{0}}{\lambda_{\min }(Q)}, c_{3}=\lambda_{\min }(R), c_{4}=\frac{\|W B\| \varepsilon_{p_{0}}}{\lambda_{\min }(R)}, c_{5}=\lambda_{\min }\left(B^{\top} A_{m}^{-\top} Q A_{m}^{-1} B\right)\|z(x, r)\|^{2}, c_{6}=$ $\frac{\left\|B^{\top} P A_{m}^{-1} B\right\| K_{0}}{\lambda_{\min }\left(B^{\top} A_{m}^{-\top} Q A_{m}^{-1} B\right)}, c_{7}=\lambda_{\min }\left(A_{m}^{-\top} R A_{m}^{-1}\right)\|\Psi(x, r)\|^{2}$, and $c_{8}=\frac{\eta\left\|W A_{m}^{-1}\right\|\|\Psi(x, r)\|^{2} B_{0}^{2} \Omega_{0}+\gamma_{w}^{-1} \dot{w}_{0}}{\eta \lambda_{\min }\left(A_{m}^{-\top} R A_{m}^{-1}\right)\|\Psi(x, r)\|^{2} B_{0}^{2}}$. Then

$$
\begin{aligned}
\dot{V} \leq-c_{1}\left(\|e\|-c_{2}\right)^{2}+c_{1} c_{2}^{2}- & c_{3}\left(\left\|e_{p}\right\|-c_{4}\right)^{2}+c_{3} c_{4}^{2} \\
& -v c_{5}\|\hat{\Lambda}\|^{2}\left(\|\tilde{K}\|-c_{6}\right)^{2}+v c_{5} c_{6}^{2}\|\hat{\Lambda}\|^{2}-\eta c_{7}\|\bar{B}\|^{2}\left(\|\tilde{\Omega}\|-c_{8}\right)^{2}+\eta c_{7} c_{8}^{2}\|\bar{B}\|^{2}
\end{aligned}
$$

Note that

$$
\begin{gathered}
\|\hat{\Lambda}\| \leq\|\Omega\| \Rightarrow\|\tilde{\Lambda}\| \leq\|\tilde{\Omega}\| \\
\|\hat{\Lambda}\|^{2}=\|\Lambda+\tilde{\Lambda}\|^{2} \leq\|\Lambda\|^{2}+2\|\Lambda\|\|\tilde{\Lambda}\|+\|\tilde{\Lambda}\|^{2} \leq\|\Lambda\|^{2}+2\|\Lambda\|\|\tilde{\Omega}\|+\|\tilde{\Omega}\|^{2} \\
\|\bar{B}\|^{2}=\left\|\bar{B}^{*}+\tilde{B}\right\|^{2} \leq\left\|\bar{B}^{*}\right\|^{2}+2\left\|\bar{B}^{*}\right\|\|\tilde{B}\|+\|\tilde{B}\|^{2}=\left\|\bar{B}^{*}\right\|^{2}+2\left\|\bar{B}^{*}\right\|\|B\|\|\tilde{\Lambda}\|+\|B\|^{2}\|\tilde{\Lambda}\|^{2} \\
\leq\left\|\bar{B}^{*}\right\|^{2}+2\left\|\bar{B}^{*}\right\|\|B\|\|\tilde{\Omega}\|+\|B\|^{2}\|\tilde{\Omega}\|^{2}
\end{gathered}
$$

where $\left\|\bar{B}^{*}\right\|=\left[\begin{array}{lll}B \Lambda & B & I\end{array}\right] \in \mathbb{R}^{n} \times \mathbb{R}^{2 m+n}$. 
Thus, $\dot{V}\left(e, e_{p}, \tilde{K}, \tilde{\Omega}\right) \leq 0$ outside a compact set $\mathscr{S}$ defined as

$$
\begin{aligned}
\mathscr{S}=\left\{\left(e(t), e_{p}(t), \tilde{K}(t), \tilde{\Omega}(t)\right): c_{1}\left(\|e\|-c_{2}\right)^{2}\right. & +c_{3}\left(\left\|e_{p}\right\|-c_{4}\right)^{2}+v c_{5} \Lambda_{0}^{2}\left(\|\tilde{K}\|-c_{6}\right)^{2} \\
& \left.+\eta c_{7} B_{0}^{2}\left(\|\tilde{\Omega}\|-c_{8}\right)^{2} \leq c_{1} c_{2}^{2}+c_{3} c_{4}^{2}+v c_{5} c_{6}^{2} \Lambda_{0}^{2}+\eta c_{7} c_{8}^{2} B_{0}^{2}\right\}
\end{aligned}
$$

where $\Lambda_{0}^{2}=\|\Lambda\|^{2}+2\|\Lambda\| c_{8}+c_{8}^{2}$ and $B_{0}^{2}=\left\|\bar{B}^{*}\right\|^{2}+2\left\|\bar{B}^{*}\right\|\|B\| c_{8}+\|B\|^{2} c_{8}^{2}$.

This implies

$$
\begin{gathered}
\|e\| \geq c_{2}+\sqrt{c_{2}^{2}+\frac{c_{3} c_{4}^{2}+v c_{5} c_{6}^{2} \Lambda_{0}^{2}+\eta c_{7} c_{8}^{2} B_{0}^{2}}{c_{1}}}=r \\
\left\|e_{p}\right\| \geq c_{4}+\sqrt{c_{4}^{2}+\frac{c_{1} c_{2}^{2}+v c_{5} c_{6}^{2} \Lambda_{0}^{2}+\eta c_{7} c_{8}^{2} B_{0}^{2}}{c_{3}}}=p \\
\|\tilde{K}\| \geq c_{6}+\sqrt{c_{6}^{2}+\frac{c_{1} c_{2}^{2}+c_{3} c_{4}^{2}+\eta c_{7} c_{8}^{2} B_{0}^{2}}{v c_{5} \Lambda_{0}^{2}}}=\alpha \\
\|\tilde{\Omega}\| \geq c_{8}+\sqrt{c_{8}^{2}+\frac{c_{1} c_{2}^{2}+c_{3} c_{4}^{2}+v c_{5} c_{6}^{2} \Lambda_{0}^{2}}{\eta c_{7} B_{0}^{2}}}=\beta
\end{gathered}
$$

Note that the bounds are dependent on $\|z(x, r)\|$ and $\|\Psi(x, r)\|$. Then it can be shown that there exist $z_{0}$ and $\Psi_{0}$ such that $\|z(x, r)\| \leq z_{0}$ and $\|\Psi(x, r)\| \leq \Psi_{0}$ for any $0<v<v_{\max }$ and $0<\eta<\eta_{\max }$ that satisfy the following inequalities:

$$
\begin{aligned}
\varphi\left(\|x\|,\left\|x_{m}\right\|, Q, v, w_{0}, \varepsilon_{0}, \Lambda_{0}, K_{0}\right)=-c_{1}\|x\|^{2}+2 & \left(c_{1} c_{2}+\lambda_{\max }(Q)\left\|x_{m}\right\|\right)\|x\|+2 c_{1} c_{2}\left\|x_{m}\right\|-c_{1}\left\|x_{m}\right\|^{2} \\
& +c_{3} c_{4}^{2}+v c_{5}(\|z(x, r)\|) c_{6}^{2} \Lambda_{0}^{2}+\eta c_{7}(\|\Psi(x, r)\|) c_{8}^{2} B_{0}^{2} \leq 0 \\
\phi\left(\left\|x_{p}\right\|,\left\|x_{m}\right\|, R, \eta, \dot{w}_{0}, \varepsilon_{p_{0}}, B_{0}, \Omega_{0}\right)=-c_{3}\left\|x_{p}\right\|^{2} & +2\left(c_{3} c_{4}+\lambda_{\max }(R)\|x\|\right)\left\|x_{p}\right\|+2 c_{3} c_{4}\|x\|-c_{3}\|x\|^{2} \\
& +c_{1} c_{2}^{2}+v c_{5}(\|z(x, r)\|) c_{6}^{2} \Lambda_{0}^{2}+\eta c_{7}(\|\Psi(x, r)\|) c_{8}^{2} B_{0}^{2} \leq 0
\end{aligned}
$$

Therefore, the closed-loop system is uniformly ultimately bounded with the following ultimate bounds:

$$
\begin{aligned}
& \|e\| \leq \sqrt{\frac{\lambda_{\max }(P) r^{2}+\lambda_{\max }(W) p^{2}+\lambda_{\max }\left(\Gamma_{x}^{-1}, \Gamma_{r}^{-1}, \Gamma_{\Theta}^{-1}\right) \alpha^{2}+\lambda_{\max }\left(\Gamma_{\Theta}^{-1}, \Gamma_{\Lambda}^{-1}, \gamma_{w}^{-1}\right) \beta^{2}}{\lambda_{\min }(P)}} \\
& \left\|e_{p}\right\| \leq \sqrt{\frac{\lambda_{\max }(P) r^{2}+\lambda_{\max }(W) p^{2}+\lambda_{\max }\left(\Gamma_{x}^{-1}, \Gamma_{r}^{-1}, \Gamma_{\Theta}^{-1}\right) \alpha^{2}+\lambda_{\max }\left(\Gamma_{\Theta}^{-1}, \Gamma_{\Lambda}^{-1}, \gamma_{w}^{-1}\right) \beta^{2}}{\lambda_{\min }(W)}} \\
& \|\tilde{K}\| \leq \sqrt{\frac{\lambda_{\max }(P) r^{2}+\lambda_{\max }(W) p^{2}+\lambda_{\max }\left(\Gamma_{x}^{-1}, \Gamma_{r}^{-1}, \Gamma_{\Theta}^{-1}\right) \alpha^{2}+\lambda_{\max }\left(\Gamma_{\Theta}^{-1}, \Gamma_{\Lambda}^{-1}, \gamma_{w}^{-1}\right) \beta^{2}}{\lambda_{\min }\left(\Gamma_{x}^{-1}, \Gamma_{r}^{-1}, \Gamma_{\Theta}^{-1}\right)}} \\
& \|\tilde{\Omega}\| \leq \sqrt{\frac{\lambda_{\max }(P) r^{2}+\lambda_{\max }(W) p^{2}+\lambda_{\max }\left(\Gamma_{x}^{-1}, \Gamma_{r}^{-1}, \Gamma_{\Theta}^{-1}\right) \alpha^{2}+\lambda_{\max }\left(\Gamma_{\Theta}^{-1}, \Gamma_{\Lambda}^{-1}, \gamma_{w}^{-1}\right) \beta^{2}}{\lambda_{\min }\left(\Gamma_{\Theta}^{-1}, \Gamma_{\Lambda}^{-1}, \gamma_{w}^{-1}\right)}}
\end{aligned}
$$

Example: Consider a first-order SISO plant

$$
\dot{x}=a x+b \lambda\left[u\left(t-t_{d}\right)+\theta^{*} x^{2}\right]+w
$$

where $a=-1$ and $b=1$ are known, $\lambda=-1$ and $\theta^{*}=0.1$ are unknown, $t_{d}=0.2 \mathrm{sec}$ is a known time delay, and $w(t)=0.01(\sin t+\cos 2 t)$ 
The reference model is given by

$$
\dot{x}_{m}=a_{m} x_{m}+b_{m} r
$$

where $a_{m}=-2, b_{m}=2$, and $r(t)=\sin t$.

The nominal control input effectiveness is equal to unity, i.e., $\lambda^{*}=1$. So, $\lambda=-1$ represents a full control reversal.

The adaptive controller is designed as

$$
u=k_{x}(t) x+k_{r} r(t)-\theta(t) x^{2}
$$

where $k_{x}(t), k_{r}(t)$, and $\theta(t)$ are computed by the following bi-objective optimal control modification adaptive laws:

$$
\begin{gathered}
\dot{k}_{x}=\gamma_{x} x\left(e+v a_{m}^{-1} u b \hat{\lambda}\right) b \hat{\lambda} \\
\dot{k}_{r}=\gamma_{r} r\left(e+v a_{m}^{-1} u b \hat{\lambda}\right) b \hat{\lambda} \\
\dot{\theta}=-\gamma_{\theta} x^{2}\left[e+e_{p}-v a_{m}^{-1}\left(2 \theta x^{2} b \hat{\lambda}+\hat{w}\right)\right] b \hat{\lambda} \\
\dot{\hat{\lambda}}=-\gamma_{\lambda}\left(u+\theta x^{2}\right)\left\{e_{p}-v a_{m}^{-1}\left[\left(u+2 \theta x^{2}\right) b \hat{\lambda}+\hat{w}\right]\right\} b \\
\dot{\hat{w}}=-\gamma_{w}\left\{e_{p}-v a_{m}^{-1}\left[\left(u+2 \theta x^{2}\right) b \hat{\lambda}+\hat{w}\right]\right\}
\end{gathered}
$$

where the predictor error $e_{p}(t)=\hat{x}(t)-x(t)$ is computed from the predictor model

$$
\dot{\hat{x}}=a_{m} \hat{x}+\left(a-a_{m}\right) x+b \hat{\lambda}\left[u\left(t-t_{d}\right)+\theta x^{2}\right]+\hat{w}
$$

The initial conditions are $k_{x}(0)=k_{x}^{*}, k_{r}(0)=k_{r}^{*}, \theta(0)=0, \hat{\lambda}(0)=\lambda^{*}, \hat{w}(0)=0$. The adaptive gains are chosen to be $\gamma_{x}=\gamma_{r}=\gamma_{\theta}=\gamma_{\lambda}=\gamma_{w}=10$, and the modification parameters are chosen to be $v=0.1$ and $\eta=0.01$.

The closed-loop response with $r(t)=\sin t$ for $t \in[0,100]$ is shown in Figure 2. It can be seen that $x(t)$ eventually tracks $x_{m}(t)$, but the two signals are initially $180^{\circ}$ out of phase due to the control reversal. The signal $\hat{x}(t)$ approximates $x(t)$ very well.
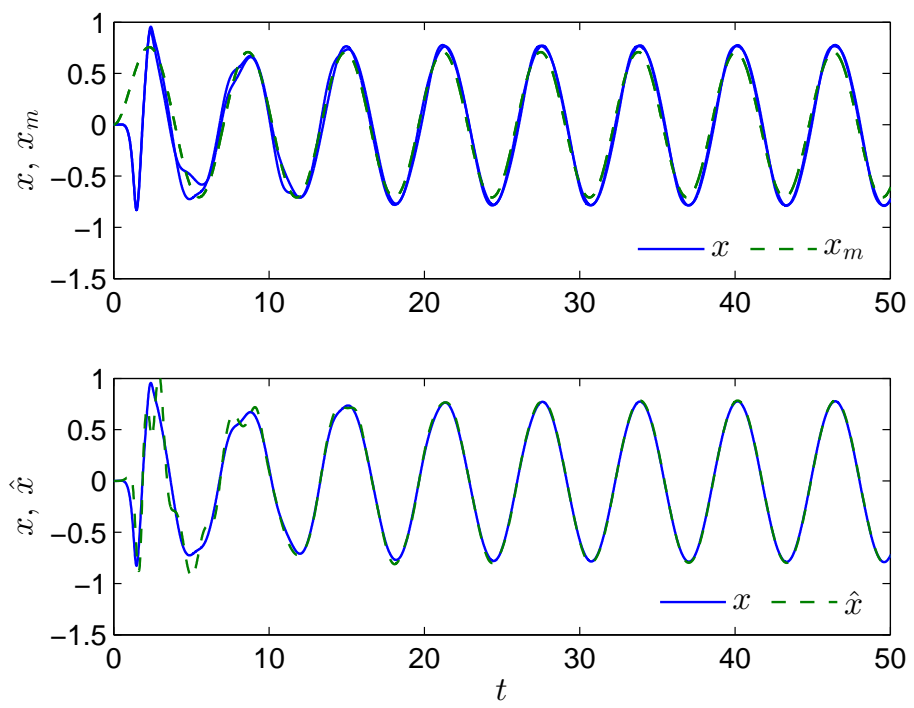

Figure $2-x(t), \hat{x}(t)$, and $x_{m}(t)$

The control parameters $k_{x}(t), k_{r}(t)$, and $\theta(t)$ are shown in Figure 3. These parameters appear to converge to their ideal values. The convergence is facilitated by having a persistently exciting reference command $\operatorname{signal} r(t)=\sin t$. 

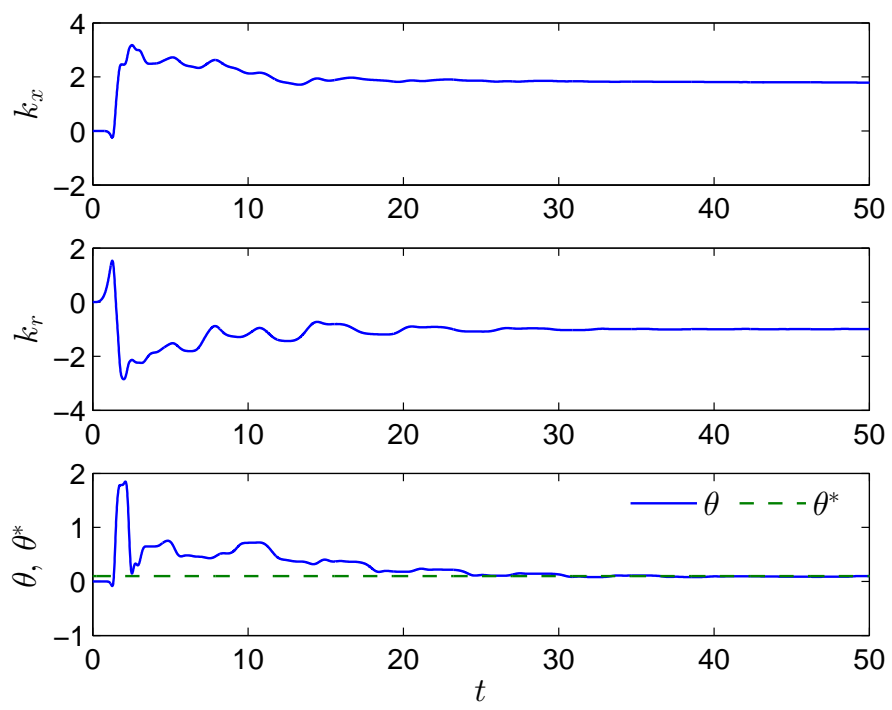

Figure $3-k_{x}(t), k_{r}(t)$, and $\theta(t)$

The control input uncertainty $\hat{\lambda}(t)$ and unmatched disturbance $\hat{w}(t)$ are estimated as shown in Figure 4.
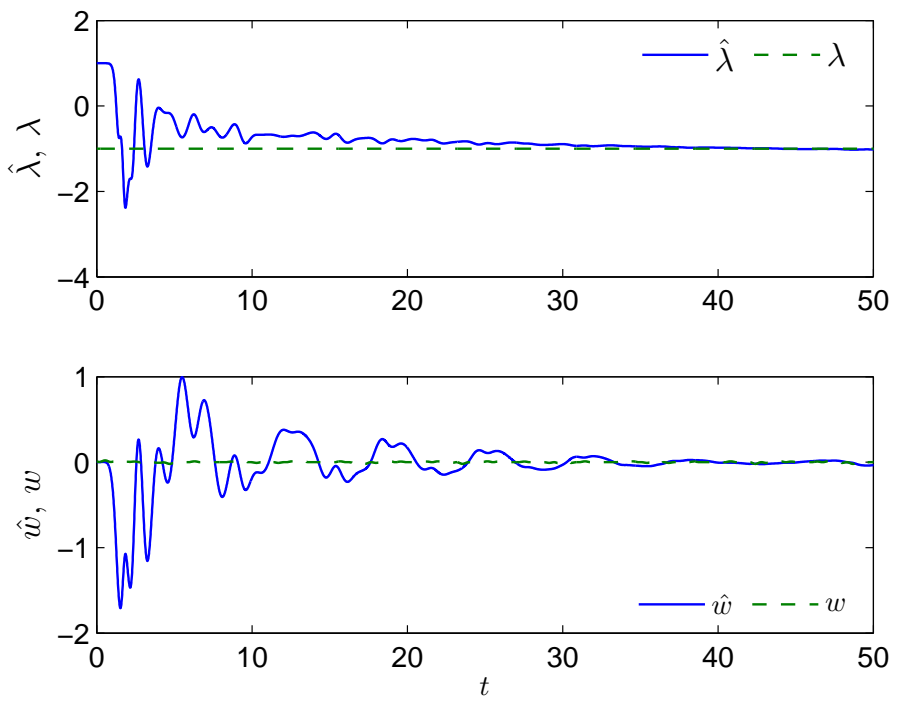

Figure $4-\hat{\lambda}(t)$ and $\hat{w}(t)$

Overall, the bi-objective optimal control modification adaptive laws demonstrate good tracking performance.

Remark: Consider an alternate representation of the plant in Eq. (1)

$$
\dot{x}=A x+B\left[u+\Theta^{* \top} \Phi(x)\right]+w
$$

where $B$ is unknown.

Then, the bi-objective optimal control modification adaptive laws can be recast as

$$
\begin{aligned}
& \dot{K}_{x}^{\top}=\Gamma_{x} x\left(e^{\top} P+v u^{\top} \hat{B}^{\top} P A_{m}^{-1}\right) \hat{B} \\
& \dot{K}_{r}^{\top}=\Gamma_{r} r\left(e^{\top} P+v u^{\top} \hat{B}^{\top} P A_{m}^{-1}\right) \hat{B}
\end{aligned}
$$




$$
\begin{gathered}
\dot{\Theta}=-\Gamma_{\Theta} \Phi(x)\left(e^{\top} P+v u^{\top} \hat{B}^{\top} P A_{m}^{-1}+e_{p}^{\top} W-\eta\left\{\left[u+2 \Theta^{\top} \Phi(x)\right]^{\top} \hat{B}^{\top}+\hat{w}^{\top}\right\} W A_{m}^{-1}\right) \hat{B} \\
\dot{\hat{B}}^{\top}=-\Gamma_{\Lambda}\left[u+\Theta^{\top} \Phi(x)\right]\left(e_{p}^{\top} W-\eta\left\{\left[u+2 \Theta^{\top} \Phi(x)\right]^{\top} \hat{B}^{\top}+\hat{w}^{\top}\right\} W A_{m}^{-1}\right) \\
\dot{\hat{w}}^{\top}=-\gamma_{w}\left(e_{p}^{\top} W-\eta\left\{\left[u+2 \Theta^{\top} \Phi(x)\right]^{\top} \hat{B}^{\top}+\hat{w}^{\top}\right\} W A_{m}^{-1}\right)
\end{gathered}
$$

where $\hat{B}$ is the estimate of $B$.

\section{Flight Control Simulation}

Consider a longitudinal pitch dynamic model of an aircraft

$$
\begin{aligned}
{\left[\begin{array}{ccc}
m V+\frac{C_{L_{\dot{\alpha}}} \bar{q} S \bar{c}}{2 V} & 0 & 0 \\
0 & 1 & 0 \\
-\frac{C_{m_{\dot{\alpha}}} \bar{q} S \bar{c}^{2}}{2 V} & 0 & I_{y y}
\end{array}\right]\left[\begin{array}{c}
\dot{\alpha} \\
\dot{\theta} \\
\dot{q}
\end{array}\right]=} & {\left[\begin{array}{ccc}
m g \gamma-C_{L_{\alpha}} \bar{q} S & -m g \gamma & m V-\frac{C_{L_{q}} \bar{q} S \bar{c}}{2 V} \\
0 & 0 & 1 \\
C_{m_{\alpha}} \bar{q} S \bar{c} & 0 & \frac{C_{m_{q}} \bar{q} S \bar{c}^{2}}{2 V}
\end{array}\right]\left[\begin{array}{c}
\alpha \\
\theta \\
q
\end{array}\right] } \\
& +\lambda\left[\begin{array}{c}
-C_{L_{\delta_{e}}} \\
0 \\
C_{m_{\delta_{e}}}
\end{array}\right]\left(\delta_{e}\left(t-t_{d}\right)+\left[\begin{array}{lll}
\theta_{\alpha}^{*} & 0 & \theta_{q}^{*}
\end{array}\right]\left[\begin{array}{l}
\alpha \\
\theta \\
q
\end{array}\right]\right)+\left[\begin{array}{c}
w_{\alpha} \\
w_{\theta} \\
w_{q}
\end{array}\right]
\end{aligned}
$$

where $t_{d}=50 \mathrm{msec}$ is a time delay introduced to account for unmodeled dynamics, and $\lambda \in[0,1]$ is the control input effectiveness, normally equal to 1 .

A numerical model for a full-scale generic transport model (GTM) at Mach 0.8 and 30,000 $\mathrm{ft}$ with the flight path angle $\gamma=0$ is given by

$$
\left[\begin{array}{c}
\dot{\alpha} \\
\dot{\theta} \\
\dot{q}
\end{array}\right]=\underbrace{\left[\begin{array}{ccc}
-0.7018 & 0 & 0.9761 \\
0 & 0 & 1 \\
-2.6923 & 0 & -0.7322
\end{array}\right]}_{A}\left[\begin{array}{c}
\alpha \\
\theta \\
q
\end{array}\right]+\underbrace{\left[\begin{array}{c}
-0.0573 \\
0 \\
-3.5352
\end{array}\right]}_{B}\left(\delta_{e}\left(t-t_{d}\right)+\left[\begin{array}{lll}
\theta_{\alpha}^{*} & 0 & \theta_{q}^{*}
\end{array}\right]\left[\begin{array}{c}
\alpha \\
\theta \\
q
\end{array}\right]\right)+\left[\begin{array}{l}
w_{\alpha} \\
w_{\theta} \\
w_{q}
\end{array}\right]
$$

where the disturbances are given by

$$
\left[\begin{array}{l}
w_{\alpha} \\
w_{\theta} \\
w_{q}
\end{array}\right]=\left[\begin{array}{c}
0.01 \sin t-0.05 e^{-0.1 t} \cos 2 t \\
-0.01 \cos 4 t \\
0.02 e^{-0.5 t} \sin 3 t-0.03 \cos t \sin 2 t
\end{array}\right]
$$

A desired reference model of the pitch attitude is given by

$$
\ddot{\theta}_{m}(t)+2 \zeta \omega_{n} \dot{\theta}_{m}(t)+\omega_{n}^{2} \theta_{m}(t)=\omega_{n}^{2} r(t)
$$

where $\zeta=0.85$ and $\omega_{n}=1.5 \mathrm{rad} / \mathrm{sec}$ are chosen to give a desired handling characteristic.

Let $x=\left[\begin{array}{ccc}\alpha & \theta & q\end{array}\right]^{\top}, u=\delta_{e}, \Theta^{* \top}=\left[\begin{array}{ccc}\theta_{\alpha}^{*} & 0 & \theta_{q}^{*}\end{array}\right]=\left[\begin{array}{ccc}0.4 & 0 & -0.3071\end{array}\right]$, and $\lambda=0.5$. The parametric uncertainty $\Theta^{*}$ and the control input uncertainty $\lambda$ result in short-period mode damping ratio of 0.2418 , which is almost half of nominal short-period mode damping ratio of 0.4045. A nominal controller is designed with $K_{x}=\frac{1}{b_{3}}\left[\begin{array}{lll}a_{31} & \omega_{n}^{2} & 2 \zeta \omega_{n}+a_{33}\end{array}\right]=\left[\begin{array}{lll}-0.7616 & 0.6365 & 0.5142\end{array}\right]$ and $k_{r}=\frac{1}{b_{3}} \omega_{n}^{2}=-0.6365$. The closed-loop eigenvalues of the ideal plant are -0.6582 and $-1.2750 \pm 0.7902 i$. The nominal closed-loop plant is then chosen to be the reference model as

$$
\underbrace{\left[\begin{array}{c}
\dot{\alpha}_{m} \\
\dot{\theta}_{m} \\
\dot{q}_{m}
\end{array}\right]}_{\dot{x}_{m}}=\underbrace{\left[\begin{array}{ccc}
-0.6582 & -0.0365 & 0.9466 \\
0 & 0 & 1 \\
0 & -2.2500 & -2.5500
\end{array}\right]}_{A_{m}} \underbrace{\left[\begin{array}{c}
\alpha_{m} \\
\theta_{m} \\
q_{m}
\end{array}\right]}_{x_{m}}+\underbrace{\left[\begin{array}{c}
0.0365 \\
0 \\
2.2500
\end{array}\right]}_{B_{m}} r
$$


If the adaptive controller is given by

$$
u=u_{n o m}+u_{a d}
$$

where

$$
\begin{gathered}
u_{\text {nom }}=K_{x} x+k_{r} r \\
u_{a d}=\Delta K_{x}(t) x+\Delta k_{r}(t) r-\Theta^{\top}(t) x
\end{gathered}
$$

then, $\Delta K_{x}(t), \Delta k_{r}(t)$, and $\Theta^{\top}(t)$ are computed from the following bi-objective optimal control modification adaptive laws:

$$
\begin{gathered}
\Delta \dot{K}_{x}^{\top}=\Gamma_{K_{x}} x\left(e^{\top} P+v u_{a d}^{\top} \hat{\Lambda}^{\top} B^{\top} P A_{m}^{-1}\right) B \hat{\Lambda} \\
\Delta \dot{k}_{r}=\gamma_{k_{r}} r\left(e^{\top} P+v u_{a d}^{\top} \hat{\Lambda}^{\top} B^{\top} P A_{m}^{-1}\right) B \hat{\Lambda} \\
\dot{\Theta}=-\Gamma_{\Theta} x\left(e^{\top} P+v u_{a d}^{\top} \hat{\Lambda}^{\top} B^{\top} P A_{m}^{-1}+e_{p}^{\top} W-\eta\left\{\left[u+2 \Theta^{\top} \Phi(x)\right]^{\top} \hat{B}^{\top}+\hat{w}^{\top}\right\} W A_{m}^{-1}\right) B \hat{\Lambda} \\
\dot{\hat{\Lambda}}^{\top}=-\Gamma_{\Lambda}\left[u+\Theta^{\top} x\right]\left(e_{p}^{\top} W-\eta\left\{\left[u+2 \Theta^{\top} \Phi(x)\right]^{\top} \hat{\Lambda}^{\top} B^{\top}+\hat{w}^{\top}\right\} W A_{m}^{-1}\right) B \\
\dot{\hat{w}}^{\top}=-\gamma_{w}\left(e_{p}^{\top} W-\eta\left\{\left[u+2 \Theta^{\top} \Phi(x)\right]^{\top} \hat{\Lambda}^{\top} B^{\top}+\hat{w}^{\top}\right\} W A_{m}^{-1}\right)
\end{gathered}
$$

These adaptive laws are alternative expressions to those of Eqs. (29) to (33) and (83) to (87).

Figure 5 shows the aircraft response due to the baseline controller. With no adaptation, the closed-loop plant becomes unstable after about $23 \mathrm{sec}$. Figure 6 is the plot of the aircraft response with the standard MRAC for the adaptive gains $\Gamma_{K_{x}} x=\Gamma_{\Theta}=\Gamma_{\Lambda}=50 I$ and $\gamma_{w}=50$. The command tracking has improved considerably. However, there is a large initial transient in the pitch rate response as well as high frequency oscillations.

Figure 7 shows the aircraft response with the bi-objective MRAC when $v=\eta=0$. The closed-loop becomes unstable after $9 \mathrm{sec}$. The instability of the adaptive laws is consistent with the theory which shows that $\eta$ cannot be zero when an external disturbance $w(t)$ exists due to the term $c_{8}$ in the stability theorem. Moreover, it is also consistent with the MRAC theory which shows that the standard MRAC generally exhibits a parameter drift in the presence of a disturbance. To prevent parameter drift, the disturbance estimate $\hat{w}(t)$ must be bounded by setting $\eta>0$. Alternatively, if the disturbance $w(t)$ is not estimated by setting $\gamma_{w}=0$, then stability of the bi-objective MRAC will be restored since the term $c_{8}$ becomes bounded for $\eta=0$. Figure 8 illustrates this observation whereby the aircraft response becomes stable when $\gamma_{w}=0$. Comparing the aircraft response with the bi-objective MRAC with $\gamma_{w}=0$ to that with the standard MRAC, it can be seen that the bi-objective adaptation significantly reduces high frequency oscillations in the pitch rate response.

Figure 9 shows the aircraft response with the bi-objective optimal control modification for the same adaptive gains with $v=\eta=0.4$. The pitch rate response is significantly improved with virtually no noticeable large initial transient and high frequency oscillations. However, the pitch attitude tracking is somewhat degraded. This is entirely expected since the bi-objective optimal control modification adaptive laws trade tracking performance for improved robustness. Comparing the aircraft response with the bi-objective MRAC to that with the bi-objective optimal control modification, it can be seen in the pitch rate response that the bi-objective optimal control modification results in smaller initial transients and better tracking.

Figure 10 compares the elevator deflections produced by all the different controllers. The elevator deflection produced by the baseline controller is well within the position limit, but instability still occurs. All adaptive controllers with MRAC exhibit control saturation and high frequency oscillations to various extents. The elevator deflection with the standard MRAC exhibits significant high frequency oscillations and control saturation. The elevator deflection with the bi-objective MRAC with $\gamma_{w}>0$ is in full saturation before the controller goes unstable. In contrast, the bi-objective MRAC with $\gamma_{w}=0$ causes only an initial control saturation of the elevator deflection. The amplitude of the control signal then rapidly improves with small periodic transients. In contrast, the bi-objective optimal control modification produces a well-behaved control signal for the elevator deflection with no discernible saturation or high frequency oscillations. 

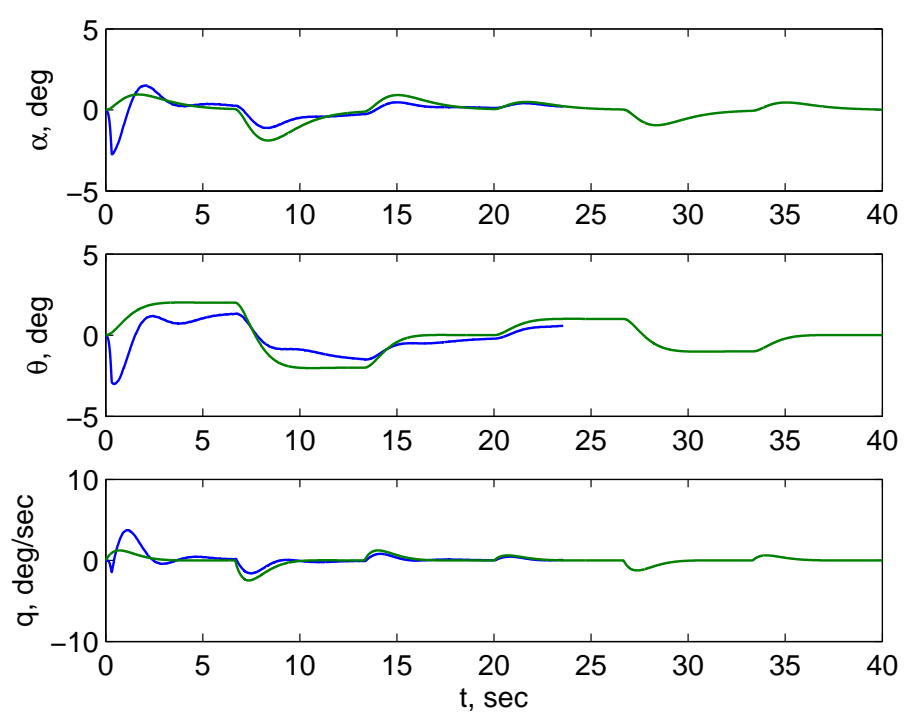

Figure 5 - Unstable Aircraft Response with Baseline Controller
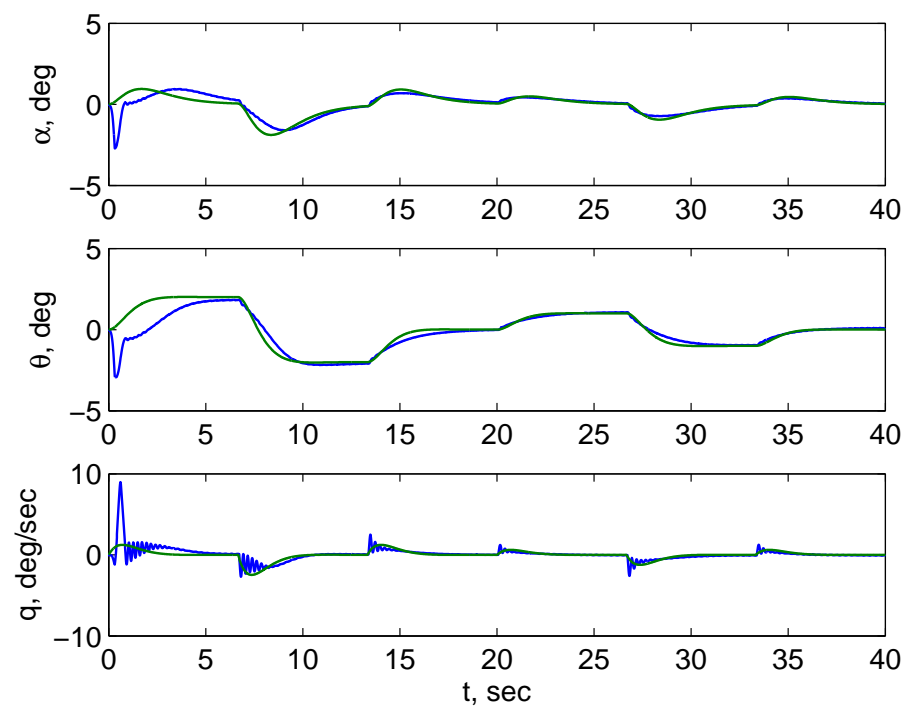

Figure 6 - Stable Aircraft Response with Standard MRAC 

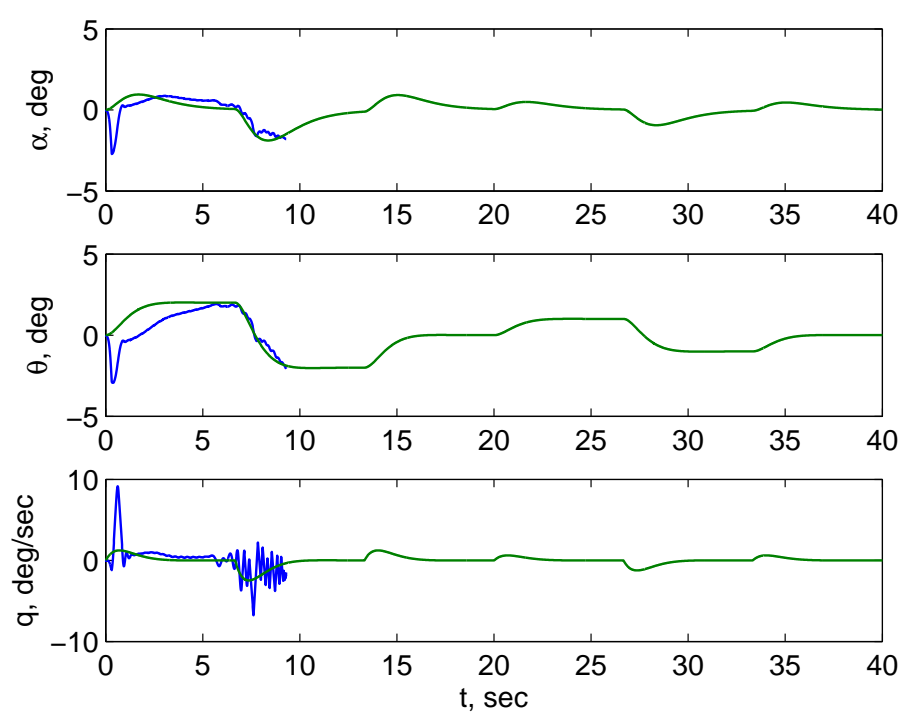

Figure 7 - Unstable Aircraft Response with Bi-Objective MRAC $\gamma_{w}>0$
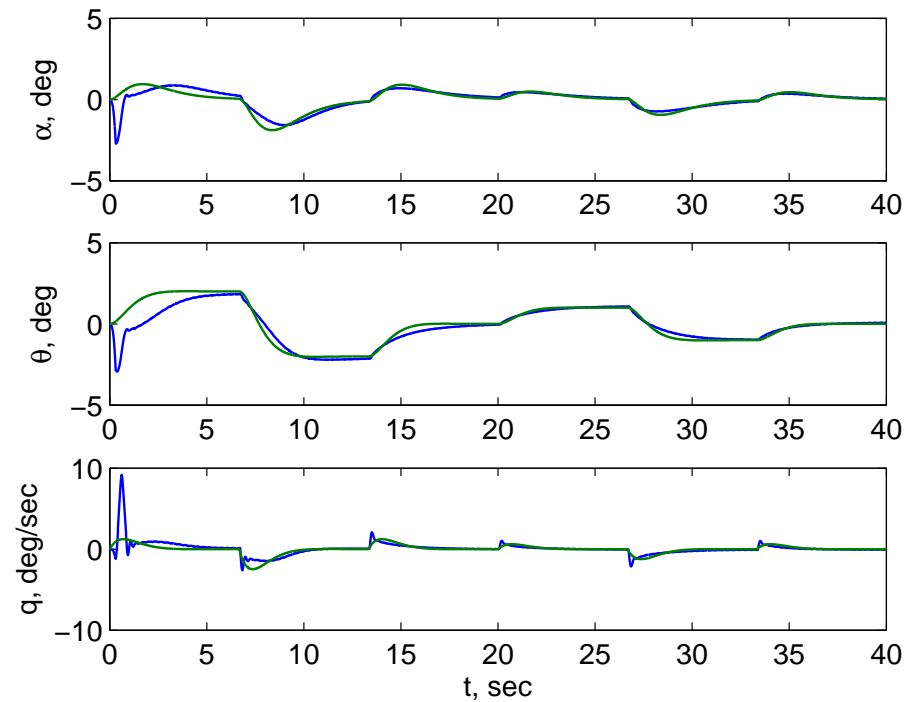

Figure 8 - Stable Aircraft Response with Bi-Objective MRAC $\gamma_{w}=0$ 

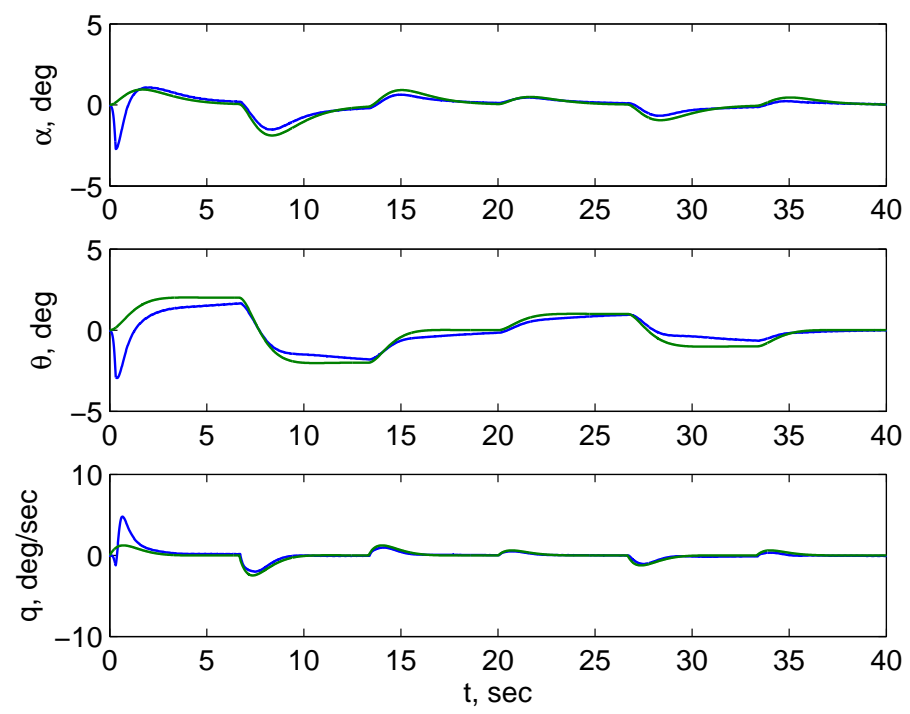

Figure 9 - Stable Aircraft Response with Bi-Objective Optimal Control Modification (OCM)
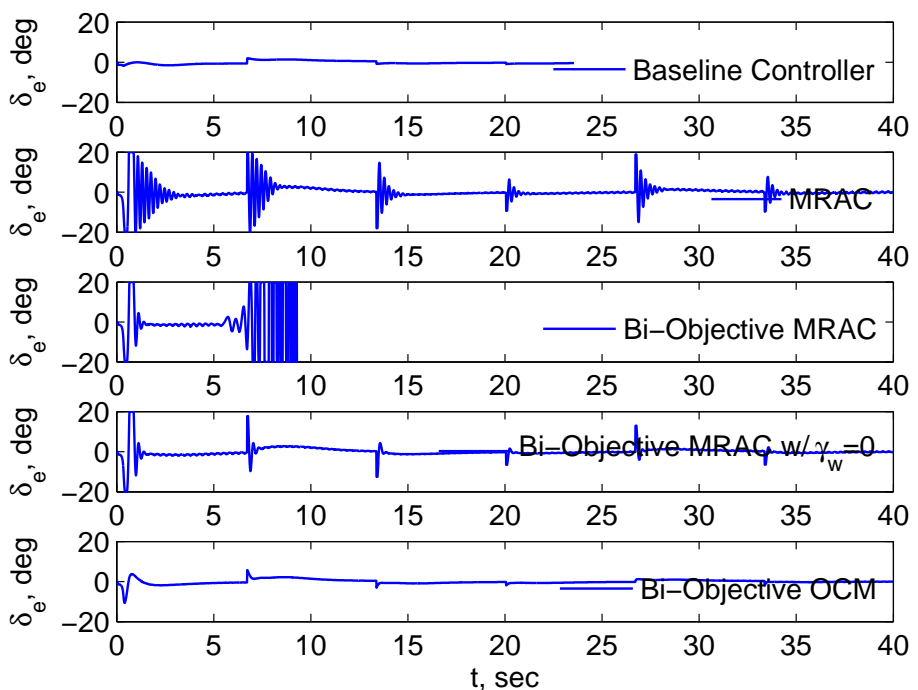

Figure 10 - Elevator Deflections

\section{Conclusions}

This study presents a new method of adaptive control for systems with input uncertainty. A parallel predictor model is constructed to relate the predictor error to the estimation error of the control effectiveness matrix. An optimal control method for a bi-objective cost function to reduce both the tracking error and predictor error simultaneously has been developed. The bi-objective optimal control modification adaptive laws enable the adaptation using both the tracking error and predictor error to improve robustness of the closed-loop systems in the presence of input uncertainty. Simulations show that the bi-objective optimal control modification adaptive laws are quite effective in maintain good tracking performance while improving robustness over the standard model-reference adaptive control.

\section{References}

${ }^{1}$ Rohrs, C.E., Valavani, L., Athans, M., and Stein, G., "Robustness of Continuous-Time Adaptive Control Algorithms in the Presence of Unmodeled Dynamics," IEEE Transactions on Automatic Control, Vol AC-30, No. 9, pp. 881-889, 1985. 
${ }^{2}$ Ioannou, P. and Kokotovic, P., "Instability Analysis and Improvement of Robustness of Adaptive Control," Automatica, Vol. 20, No. 5, 1984, pp. 583-594.

${ }^{3}$ Narendra, K. S. and Annaswamy, A. M., “A New Adaptive Law for Robust Adaptation Without Persistent Excitation,” IEEE Transactions on Automatic Control, Vol. AC-32, No. 2, pp. 134-145, 1987.

${ }^{4}$ Cao, C. and Hovakimyan, N., "Design and Analysis of a Novel $\mathscr{L}_{1}$ Adaptive Control Architecture with Guaranteed Transient Performance", IEEE Transactions on Automatic Control, Vol. 53, No. 2, pp. 586-591, 2008.

${ }^{5}$ Calise, A., Yucelen, T., Muse, J., and Yang, B., "A Loop Recovery Method for Adaptive Control," AIAA Guidance, Navigation, and Control Conference, AIAA-2009-5967, August 2009.

${ }^{6}$ Yucelen, T. and Calise, A., "A Kalman Filter Optimization Approach to Direct Adaptive Control," AIAA Guidance, Navigation, and Control Conference, AIAA-2010-7769, August 2009.

${ }^{7}$ Yucelen, T. and Calise, A., "Derivative-Free Model Reference Adaptive Control," AIAA Guidance, Navigation, and Control Conference, AIAA-2009-5858, August 2010.

${ }^{8}$ Lavretsky, E., "Combined / Composite Model Reference Adaptive Control," IEEE Transactions on Automatic Control, Vol. 54 (11), pp. 2692-2697, 2009.

${ }^{9}$ Nguyen, N., Krishnakumar, K., and Boskovic, J., ”An Optimal Control Modification to Model-Reference Adaptive Control for Fast Adaptation", AIAA Guidance, Navigation, and Control Conference, AIAA 2008-7283, August 2008.

${ }^{10}$ Jacklin, S., "Closing the Certification Gaps in Adaptive Flight Control Software," AIAA Guidance, Navigation, and Control Conference, AIAA-2008-6988, August 2008.

${ }^{11}$ Nguyen, N. and Summers, E., "On Time Delay Margin Estimation for Adaptive Control and Robust Modification Adaptive Laws," AIAA Guidance, Navigation, and Control Conference, AIAA-2011-6438, August 2011.

${ }^{12}$ Nguyen, N., "Asymptotic Linearity of Optimal Control Modification Adaptive Law with Analytical Stability Margins," AIAA Infotech@Aerospace Conference, AIAA-2010-3301, April 2010.

${ }^{13}$ Nguyen, N., Ishihara, A., Stepanyan, V., and Boskovic, J., "Singular Perturbation and Optimal Control Modification for Robust Adaptation with Slow Actuators," AIAA Guidance, Navigation, and Control Conference, AIAA-2009-5615, August 2009.

${ }^{14}$ Nguyen, N., "Verifiable Adaptive Control with Analytical Stability Margins by Optimal Control Modification," AIAA Guidance, Navigation, and Control Conference, AIAA-2010-7770, August 2010.

${ }^{15}$ Yucelen, T., Calise, A., and Nguyen, N., "Evaluation of Derivative-Free Adaptive Controller with Optimal Control Modification," AIAA Guidance, Navigation, and Control Conference, AIAA-2011-6604, August 2011.

${ }^{16}$ Nguyen, N., Tuzcu, I., Yucelen, T., and Calise, A., "Longitudinal Dynamics and Adaptive Control Application for an Aeroelastic Generic Transport Model," AIAA Atmospheric Flight Mechanics Conference, AIAA-2011-6319, August 2011.

${ }^{17}$ Reed, S., Steck, J., and Nguyen, N., "Demonstration of the Optimal Control Modification for General Aviation: Design and Simulation," AIAA Guidance, Navigation, and Control Conference, AIAA-2011-6254, August 2011.

${ }^{18}$ Burken, J., Nguyen, N., and Griffin, B., "Adaptive Flight Control Design with Optimal Control Modification for F- 18 Aircraft Model ,"AIAA Infotech@ Aerospace Conference, AIAA-2010-3364, April 2010.

${ }^{19}$ Campbell, S., Kaneshige, J., Nguyen, N., and Krishnakumar, K., "An Adaptive Control Simulation Study Using Pilot Handling Qualities Evaluations," AIAA Guidance, Navigation, and Control Conference, AIAA-2010-8013, August 2010.

${ }^{20}$ Campbell, S., Kaneshige, J., Nguyen, N., and Krishnakumar, K., "Implementation and Evaluation of Multiple Adaptive Control Technologies for a Generic Transport Aircraft Simulation,” AIAA Infotech@ Aerospace Conference, AIAA-2010-3322, April 2010.

${ }^{21}$ Schaefer, J., Hanson, C., Johnson, M., and Nguyen, N., "Handling Qualities of Model Reference Adaptive Controllers with Varying Complexity for Pitch-Roll Coupled Failures," AIAA Guidance, Navigation, and Control Conference, AIAA-2011-6453, August 2011.

${ }^{22}$ Hanson, C., Johnson, M., Schaefer, J., Nguyen, N., and Burken, J., "Handling Qualities Evaluations of Low Complexity Model Reference Adaptive Controllers for Reduced Pitch and Roll Damping Scenarios," AIAA Guidance, Navigation, and Control Conference, AIAA2011-6607, August 2011.

${ }^{23}$ Nguyen, N., Burken, J., and Hanson, C., "Optimal Control Modification Adaptive Law with Covariance Adaptive Gain Adjustment and Normalization," AIAA Guidance, Navigation, and Control Conference, AIAA-2011-6606, August 2011.

${ }^{24}$ Bryson, A. E. and Ho, Y. C., Applied Optimal Control: Optimization, Estimation, and Control, John Wiley \& Sons, Inc., 1979.

${ }^{25}$ Anderson, B. and Moore, J., Linear Optimal Control, Prentice-Hall, Inc., 1971.

${ }^{26}$ Ioannu, P. A. and Sun, J., Robust Adaptive Control, Prentice-Hall, Inc., 1996. 